\title{
The effects of flow disturbance by an oceanic island
}

\author{
by Karen J. Heywood, ${ }^{1,2}$ Eric D. Barton ${ }^{1}$ and John H. Simpson'
}

\begin{abstract}
We present CTD and ADCP surveys around the Indian Ocean coral atolls of Aldabra $\left(46^{\circ} 20^{\prime} \mathrm{E}, 9^{\circ} 25^{\prime} \mathrm{S}\right)$ and Cosmoledo $\left(47^{\circ} 30 \mathrm{E}, 9^{\circ} 45^{\prime} \mathrm{S}\right)$ during April-July 1987. These isolated, steep-sided islands situated in deep $(4000 \mathrm{~m})$ water in the South Equatorial Current provide examples of flow disturbance which are compared to laboratory studies of eddy generation by a circular cylinder in a channel.

For sufficiently strong and steady flows, a doming of the isopycnals is observed in the lee of the islands. The sea-surface temperature decreases and the salinity increases as cool, saline water is mixed from below. Just as in the case of islands in tidally dominated shelf seas, we observe an increase in the chlorophyll content of the water behind the island, indicative of enhanced phytoplankton productivity caused by the flow disturbance. This anomalous region may be associated with an eddy trapped downstream of the island. The incident flows were not strong enough to generate a vortex street of eddies spun off in a wake. When the incident flows are weak and fluctuating, no evidence for eddies is seen; and there is no doming of the isopycnals leading to low sea-surface temperature and high chlorophyll content.
\end{abstract}

\section{Introduction}

An enhancement of productivity and biomass has often been reported to occur around oceanic islands. Doty and Oguri (1956) documented enrichment around Oahu, and coined the phrase "island mass effect." Despite a common acceptance of productivity near islands (Gilmartin and Revelante, 1974) and expectation of valuable fisheries around, for example, unexploited West Indian Ocean islands (FAO, 1981), little investigation of the physical causes of this phenomenon has been undertaken. Hogg (1980a) considered the effect of seamounts and islands on ocean currents in his review, mainly of theories and laboratory experiments. Theoretical studies of an island in a steady flow (Hogg, 1972; Gordon and Hughes, 1981) and observations of island trapped waves (Hogg, 1980b) have been carried out; Wang (1982) has considered a numerical model of wind-driven flow around an ocean island. Laboratory simulations of flow over topography were reviewed by Baines and Davies (1980). Boyer and Davies

1. School of Ocean Sciences, University College of North Wales, Menai Bridge, Gwynedd, United Kingdom, LL59 SEY.

2. Present address: School of Environmental Sciences, University of East Anglia, Norwich, United Kingdom, NR4 7TJ. 
(1982) investigated the flow past a cylinder in a rotating water channel for both the $f$-plane and $\beta$-plane. Observations of flow disturbance have been limited mainly to remote sensing reports of eddy production by islands (La Violette, 1974; Fett and Rabe, 1976; Takahashi et al., 1981; Pattiaratchi et al., 1987). Hogg et al. (1978) investigated the situation around Bermuda but the lack of a significant mean flow and the presence of two Gulf Stream rings did not allow any evaluation of flow disturbance by the island. Earlier work includes Barkley's (1972) Von Karman vortex street interpretation of drift patterns downstream of a Pacific atoll. White (1973) analyzed dynamic height east of the Galapagos Islands in terms of both his own earlier Rossby wake and the Von Karman theories. In neither case was the evidence or interpretation conclusive enough to be entirely convincing. The physical oceanographic phenomena associated with islands in a flow regime therefore remain largely unknown.

During the past decade observations have been made of the physical and biological effects of islands in shelf seas dominated by a tidal regime (Simpson et al., 1982; Simpson and Tett, 1986). For both the Scilly Isles and St. Kilda, on the western shelf of the British Isles, a clear island influence on the hydrography and productivity was demonstrated. For example, the integrated column chlorophyll $a$ concentration near St. Kilda in surveys in 1984 increased from $51 \mathrm{mg} \mathrm{m}^{-2}$ in the far field to $95 \mathrm{mg} \mathrm{m}^{-2}$ near the island (from 0 to $10 \mathrm{~km}$ offshore). The enhancement of productivity was shown to be a result of locally increased vertical mixing on the flanks of the island by the tidal flows.

In this paper we present the results of an experimental study of the flow around islands in the deep ocean, where tidal flows and bottom friction are less important. Such cases are more analogous to the idealized laboratory experiments of a steady (nonoscillatory) flow around a cylinder.

\section{Theory and laboratory experiments}

The problem of flow around an oceanic island may be likened to the flow around a circular cylinder in the laboratory tank. Such studies have yielded predictions of the flow patterns in oceanic (or atmospheric) situations (see for example Batchelor, 1967). In nonrotating flow, the form of the wake behind the obstacle is determined by the Reynolds number:

$$
R e=\frac{2 U R}{\nu}
$$

where $U$ is the incident flow speed

$R$ is the radius of the cylinder

$\nu$ is the horizontal eddy diffusivity, stated by Apel (1987) to vary between $10^{2}$ and $10^{5} \mathrm{~m}^{2} \mathrm{~s}^{-1}$.

According to this theory, at low Reynolds numbers $(\sim 1)$, the flow is fully attached to the cylinder; there are no eddies or wake effects. As the Reynolds number increases 
from 1 to about 70, eddies form immediately behind the obstacle. These trapped eddies occur in contra-rotating pairs. At larger Reynolds numbers, eddies are spun off behind the cylinder in a "vortex street." If the system is rotating (rotation rate $\Omega$ ), the cyclonic eddies are more enhanced than the anticyclonic ones. Therefore for flow around an island in the southern hemisphere, predominantly clockwise eddies would be expected. These flow regimes are summarized in Figure 1. In the vortex street case, the frequency $n$ at which eddies are spun off from the island is related to the Strouhal number $S t$ (Simpson and Tett, 1986):

$$
S t=\frac{2 n R}{U}
$$

If $R e>300$, the Strouhal number is 0.21 .

More recently, laboratory experiments on rotating flows have been performed to investigate the influence of the Rossby number $(R o=U / 2 \Omega R)$ and Ekman number $\left(E k=\nu / 2 \Omega R^{2}\right)$ on eddy generation (reviewed by Baines and Davies, 1980). The Reynolds number $R e$ is the ratio of Rossby to Ekman number, therefore this theory is a generalization of the earlier one. By plotting Rossby number against Ekman number, Baines and Davies determined conditions for each flow regime to occur. This will be discussed further in Section 6.

Boyer and Davies (1982) have developed these ideas further to consider the effect of the variation of rotation across the region of interest. In geophysical terms, such flows occur on the $\beta$ plane $\left(\beta=2 \Omega \cos \Phi / R_{E}\right.$, where $R_{E}$ is the radius of the earth and $\Phi$ is latitude) while previous studies had considered flows where $\beta$ was zero ( $f$ plane). Following Merkine (1980), Boyer and Davies introduced another dimensionless parameter $\beta^{\prime}$ :

$$
\beta^{\prime}=\frac{\beta R^{2}}{U}
$$

The restriction to the $\int$ plane requires that $\beta^{\prime}$ is small; they considered flows where $\beta^{\prime}$ was of order unity. They found significant differences between $f$ plane, $\beta$ plane westward and $\beta$ plane eastward flows. The $\beta$ effect was shown to inhibit flow separation by the cylinder for eastward flow, and to enhance it for westward flow.

Few tests of these theories have been carried out for islands in deep water, although the advent of rapid surveying capabilities (Acoustic Doppler Current Profilers and the undulating CTD SeaSoar, for example) enable a more synoptic picture of the flow disturbance. Such an investigation requires a strong, steady far-field current such as that found in one of the major ocean current systems. The island should be isolated as far as possible from other islands or coastal effects, and its topography should be cylindrical or conical. In order to detect any enhancement of the primary productivity, the surrounding waters should be oligotrophic. 


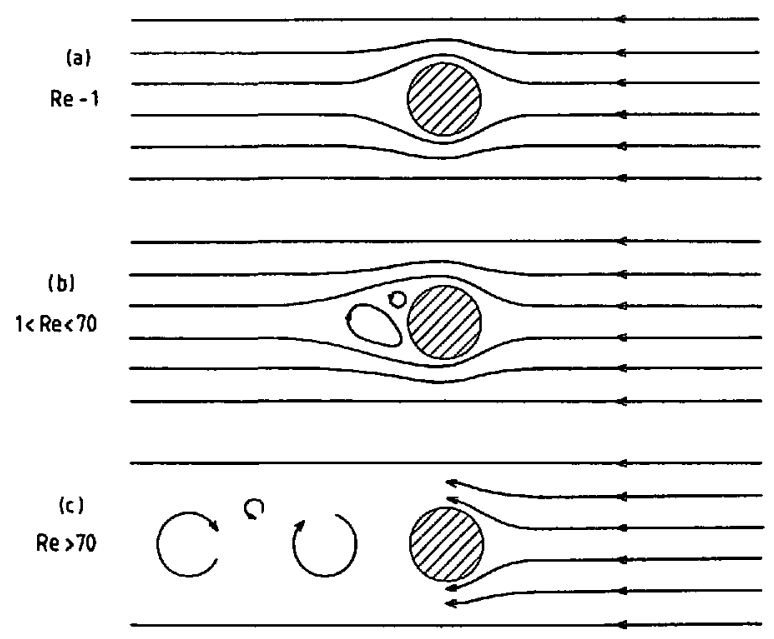

Figure 1. Flow disturbance by a circular cylinder in a homogeneous flow with rotation (analogous to the southern hemisphere). (a) $\operatorname{Re} \sim 1$, attached flow; (b) $1<\operatorname{Re}<70$, trapped eddies; (c) $R e>70$, vortex street.

An island which comes close to satisfying many of these criteria is the coral atoll of Aldabra, situated in the South Equatorial Current (SEC) in the Indian Ocean $\left(46^{\circ} 20^{\prime} \mathrm{E}, 9^{\circ} 25^{\prime} \mathrm{S}\right)$. During the months of April to July, with the onset of the austral winter, the strong SE Trade Winds force a strong surface current to the west around the northern tip of Madagascar. GEK current observations have indicated flows up to $1 \mathrm{~m} \mathrm{~s}^{-1}$ east of Aldabra (Crepon, 1964). Analysis of historical data of ship's drifts (Cutler and Swallow, 1984) predicts mean currents of a similar magnitude for 10-day averages during June and July. The results of two cruises during April to July 1987 will be described here. Our aims were to detect and identify any biological enhancement caused by the island, and to determine whether mixing or eddy generation were taking place. The flow patterns around Aldabra provide a suitable data set for comparison with laboratory experiments and the flow separation or eddy generation conditions described here.

\section{Instrumentation}

Hydrography was measured using a Neil Brown Mark III CTD. Reversing thermometers were used to check performance of the CTD temperature sensor but no evidence of deviation from laboratory calibrations was found. Salinity was corrected using determination of salinity from bottle samples using a shipboard Autosal salinometer; resulting salinities are considered accurate to 0.005 in practical units. The CTD carried a fluorometer to enable the measurement of chlorophyll concentration. On each cruise the fluorometer was calibrated against independent estimates of extracted chlorophyll content as described by Tett (1990). 
In addition to current meter moorings, current information was obtained from the ship-borne $150 \mathrm{kHz}$ RD Instruments Acoustic Doppler Current Profiler (ADCP). Such instruments require careful calibration to eliminate errors due to misalignment in the ship's hull of a few degrees. Two methods for this calibration are possible (Pollard and Read, 1989). The first requires the ADCP to be in bottom tracking mode, so the water depth must be less than about $500 \mathrm{~m}$. When close enough to Aldabra, bottom tracking was used, but because of the steeply sloping sides of the atoll, the current data have proved to be unreliable since the instrument cannot correctly determine the bottom depth. The second method requires accurate ship's navigation (such as GPS) so that the ship's speed relative to the ground is known to within a few $\mathrm{cm} \mathrm{s}^{-1}$. GPS navigation was not available for the first cruise, and only for a few hours each day on the second cruise. However calibration using 5 turns to port and 5 turns to starboard of about $90^{\circ}$ during periods of good GPS fixes (PDOP $<7$ ) has yielded estimates of the misalignment angle $\left(4.4^{\circ}\right)$ and scaling factor $\mathrm{A}(1.04)$, which have been used as the calibration.

The ADCP was operated usually with either 10 minute or 5 minute averaging intervals, giving a spatial mean over $1.5 \mathrm{~km}$ or $3 \mathrm{~km}$ if the ship steams at $10 \mathrm{knots}$. The vertical resolution is defined by our use of $8 \mathrm{~m}$ range bins. Comparison of the uppermost bin (with mean depth $11 \mathrm{~m}$ ) with those at $19 \mathrm{~m}$ and $35 \mathrm{~m}$ showed very little difference between the three depths; the current maps shown later will be for $19 \mathrm{~m}$ but $11 \mathrm{~m}$ depth currents would give the same patterns and are of similar magnitude. We observed erroneous current values caused by gyro oscillation when the ship turns, and these data are not plotted. The navigation data used to calculate absolute current velocities were derived from transit satellite fixes. By plotting an average current vector for each CTD station, uncertainties due to errors in the ship's velocity have been minimized.

\section{Data collection}

Data were collected on two cruises on the RRS Charles Darwin: Cruise 22 (11th April-8th May 1987) and Cruise 24 (15th June-14th July 1987), surveying mainly the waters surrounding Aldabra. The atoll varies in width from 10 to $15 \mathrm{~km}$; its maximum length is $35 \mathrm{~km}$ (Fig. 2). It rises steeply (about $25^{\circ}$ ) from the abyssal plain at $4000 \mathrm{~m}$. If considered as a cylinder facing a predominantly northwestward flow, a representative radius would be $10 \mathrm{~km}$. It consists of a ring of small islands enclosing a large, shallow lagoon (average depth $4 \mathrm{~m}$, approximate area $200 \times 10^{6} \mathrm{~m}^{2}$, Pugh and Rayner, 1981). The principal means of exchange of lagoon water with the open ocean is the Main Channel situated at the western end of the northern shore. This is a narrow channel through which a swift tidal current flows. Since the lagoon is large and shallow, it will be a source of warmer, saltier water every tidal cycle. Pugh and Rayner (1981) estimate that the excess temperature of the outflow water from such lagoons may be as large as $2^{\circ} \mathrm{C}$ during sunny, windless conditions. 


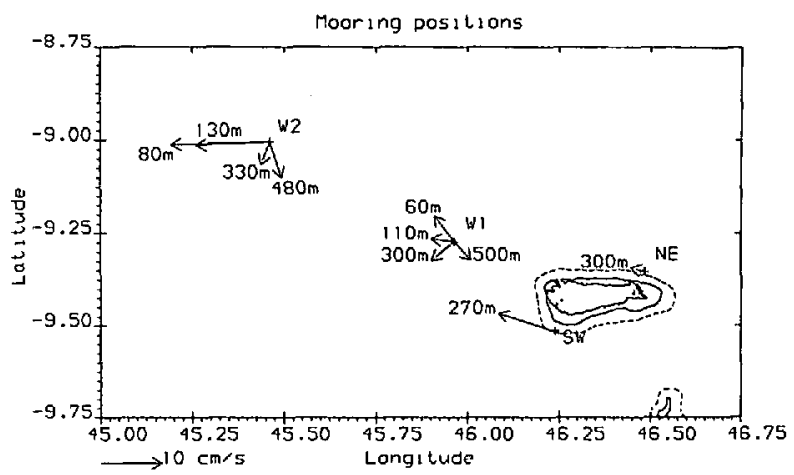

Figure 2. Mean currents at the four moorings during the 9-11 week deployments. The $1000 \mathrm{~m}$ depth contour is shown dashed.

The Cosmoledo island group, lying about $1^{\circ}$ to the east of Aldabra, is more circular in shape but slightly smaller; its radius is about $7 \mathrm{~km}$. Unlike Aldabra, Cosmoledo does not have an enclosed lagoon; most of the island is submerged except at low tide. There is therefore no tidal output of lagoon water since it is continually flushed.

According to historical data (Cutler and Swallow, 1984), the first cruise should have coincided with the beginning of the period of steady SEC incident on Aldabra. By the time of the second cruise six weeks later, a surface current of at least $1 \mathrm{~m} \mathrm{~s}^{-1}$ upstream of Aldabra was to be expected, forced by a strong and steady westward wind. To measure these currents, four moorings were deployed, two in deep water $(4000 \mathrm{~m})$ west of Aldabra, and two on the NE and SW flanks of Aldabra in $1000 \mathrm{~m}$ of water (Fig. 2). Deployed on one cruise and recovered on the other, most moorings were out for about six weeks. Shallow moorings had three Aanderaa RCM4's while deep ones had six. However several of the meters failed because of battery problems, particularly on the shallow moorings. The observed currents were weaker and more variable than the climatological atlases (e.g. Cutler and Swallow, 1984) would suggest. The mean current was indeed directed to the northwest as expected, particularly on the flanks of the island where it was constrained to flow parallel to the shore. Figure 3 shows the observed current at $80 \mathrm{~m}$ at mooring W2 (downstream) after application of a low-pass filter (Cosine-Lanczos with half power at 40 hours). The largest currents were about $0.6 \mathrm{~m} \mathrm{~s}^{-1}$; periods of strong currents lasted only a few days at most, while directions could vary by more than $90^{\circ}$ within 1 day. If the Strouhal number scaling is applied to the case of Aldabra with $0.5 \mathrm{~m} \mathrm{~s}^{-1}$ incident current, eddies would be shed approximately once every two days. Also shown are the smoothed observed wind data at the Aldabra meteorological station. Although predominantly to the northwest, the wind showed reversals and periods of fairly light winds $\left(5 \mathrm{~m} \mathrm{~s}^{-1}\right)$ for May and June.

A series of CTD surveys were undertaken around Aldabra, usually consisting of an inner ring of stations about $5 \mathrm{~km}$ from the shore (at approximately the $2,000 \mathrm{~m}$ depth contour), and an outer ring $15 \mathrm{~km}$ offshore (water depth $\sim 4000 \mathrm{~m}$ ). In addition regions 


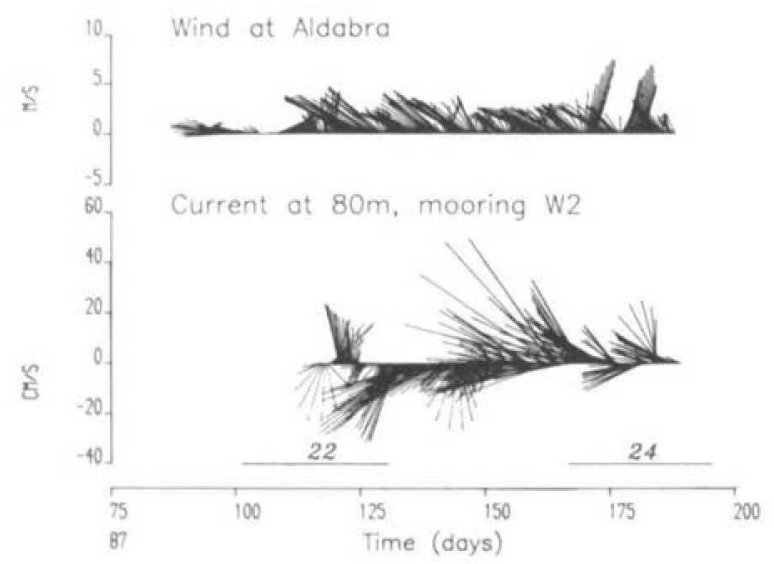

Figure 3. Low-pass-filtered wind and current velocity every six hours. Durations of Cruises 22 and 24 are marked.

downstream of the island were surveyed in conjunction with the two moorings in deep water, one and two island lengths downstream respectively. Two sets of CTD stations around Cosmoledo were completed. Most stations were to a depth of 300 or $400 \mathrm{~m}$.

We shall now present a number of case studies: two surveys around Aldabra and two around Cosmoledo (in each case, one with a strong incident current flow and one weak flow).

\section{Case studies}

a. Strong flow around Aldabra. Our first case study is a CTD survey around Aldabra during cruise 22 (18th-21 st April) during a period of fairly strong and steady incident current. The ADCP surface currents (Fig. $4 a$ ) showed a flow predominantly from the east-southeast with a magnitude of about $0.6 \mathrm{~m} \mathrm{~s}^{-1}$ outside the influence of the island. There was some acceleration of the current as it passed the flanks of the island, the maximum current $\left(0.75 \mathrm{~m} \mathrm{~s}^{-1}\right)$ being observed on the NW and SE where the two near-island moorings were deployed. Immediately in the lee of the island, the currents were variable in both magnitude and direction, but were generally weaker than the far field, defined by ADCP mapping on the approach line to the island from the east (not shown).

The CTD sections from this survey (Fig. 5) showed a doming of the isopycnals in this downstream region. The $22.0 \sigma_{t}$ contour lay at about $30 \mathrm{~m}$ upstream and on the flanks of Aldabra, whereas at the two downstream stations it reached the surface. Significantly higher chlorophyll content (calculated from fluorescence, see Tett, 1990) was observed in this downstream region. The chlorophyll was largely located between the 22.0 and $25.0 \sigma$, contours. Behind the island, therefore, a surface outcrop of chlorophyll was observed, where the isopycnals domed. Integration of the chlorophyll content of 

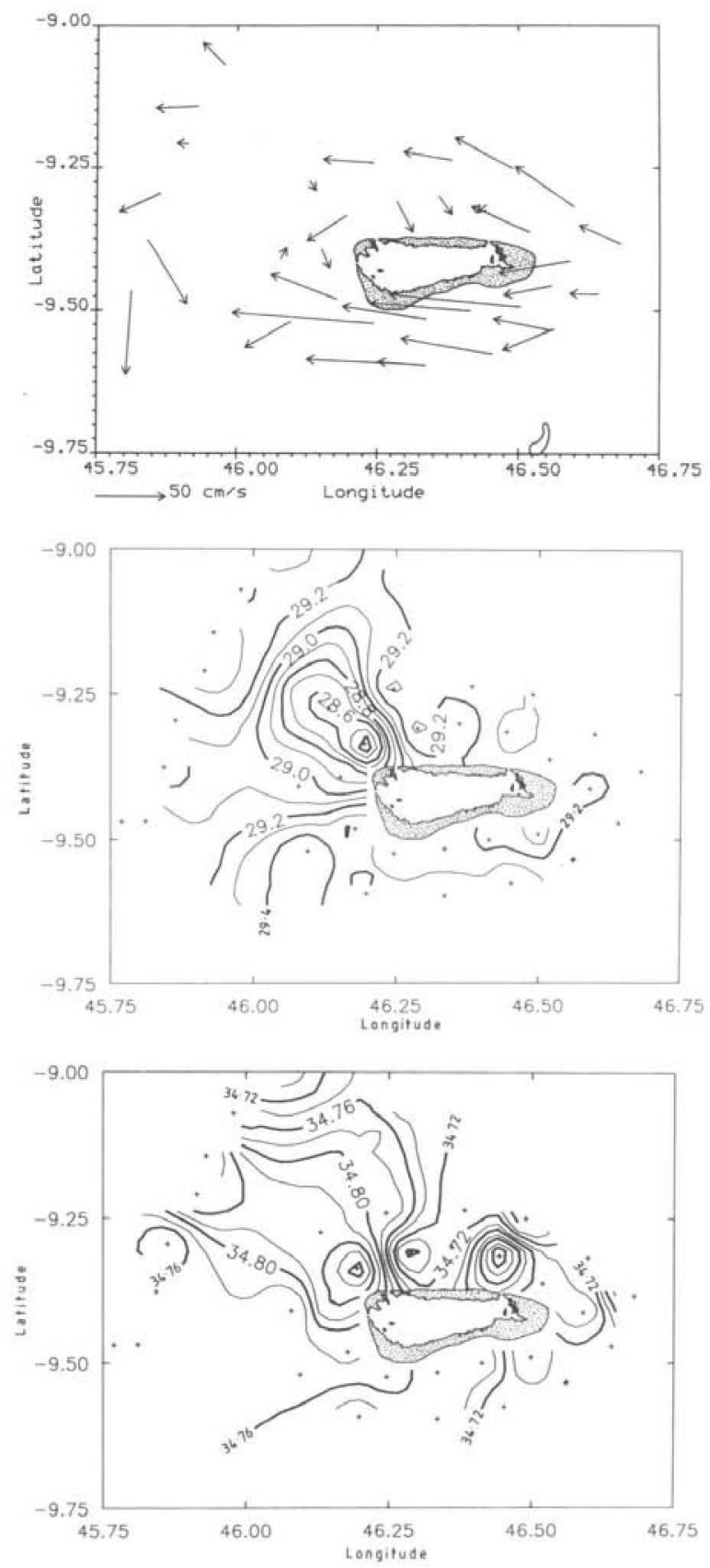

Figure 4. Oceanographic parameters during the CTD survey around Aldabra on Cruise 22, 18 April 0000-21 April 1200. CTD station positions are marked +. (a) ADCP currents at $19 \mathrm{~m}$, averages of all profiles when the ship was on station; (b) sea-surface temperature (values at $5 \mathrm{~m}$ from CTD casts); (c) sea-surface salinity (values at $5 \mathrm{~m}$ from CTD cists); (d) total integrated chlorophyll of the water column $\left(\mathrm{mg} \mathrm{m}^{-2}\right)$. 


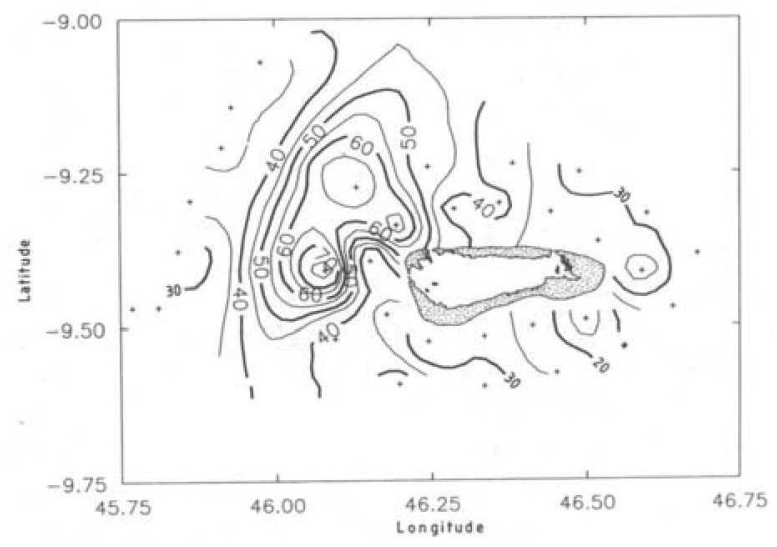

Figure 4. (Continued)

the water column (down to $200 \mathrm{~m}$; chlorophyll was negligible below about $150 \mathrm{~m}$ ) showed that this area also exhibited the highest total column chlorophyll.

The same effects of increased chlorophyll and cooler, denser water near the surface behind Aldabra were observed on a second circuit of the island immediately afterward, occupying the outer circle of CTD stations. Using both circuits a map of sea surface temperature (SST) contours (Fig. 4b) has been constructed, showing the extent of the area of lower SST behind the island. This is associated with an area of higher surface salinity (Fig. 4c), which is consistent with the upwelling of cooler, saltier water from below in a cyclonic eddy. The station at the eastern end of the northern shore also showed high surface salinity although the SST is higher. This is associated with warm saline water flowing from the lagoon through the East Channel. The integrated chlorophyll (Fig. 4d) showed a significant enhancement downstream (more than $60 \mathrm{mg} \mathrm{m}^{-2}$ at three stations). Upstream and along the flanks of the island, values were nearer to $30 \mathrm{mg} \mathrm{m}^{-3}$. The area of high ( $>60 \mathrm{mg} \mathrm{m}^{-2}$ ) productivity was estimated to be some $400 \mathrm{~km}^{2}$, approximately the same area as the atoll itself. The region coincided with the low SST (Fig. 4b) and weak currents (Fig. 4a) in the lee of Aldabra.

b. Weak flow around Aldabra. One month later, during Cruise 24, the upper ocean structure was different. A predominantly northwestward wind had been blowing (about 3-8 $\mathrm{m} \mathrm{s}^{-1}$-Fig. 3) and had increased the sensible and latent heat losses, while solar radiation decreased as winter approached. The mixed layer depth had increased from about $20 \mathrm{~m}$ to 70 or $80 \mathrm{~m}$ (Fig. 6). The phytoplankton were no longer confined to the thermocline, but instead the chlorophyll maximum occurred in the mixed layer.

Although Cruise 24 took place in the austral winter, the surface currents were weaker than during Cruise 22 . The strongest near-surface $(19 \mathrm{~m})$ currents to the northeast of Aldabra during the first CTD survey of Cruise 24 (Fig. 7a) were predominantly westward as in Cruise 22 . However the maximum current even on the 


\section{Temperature}
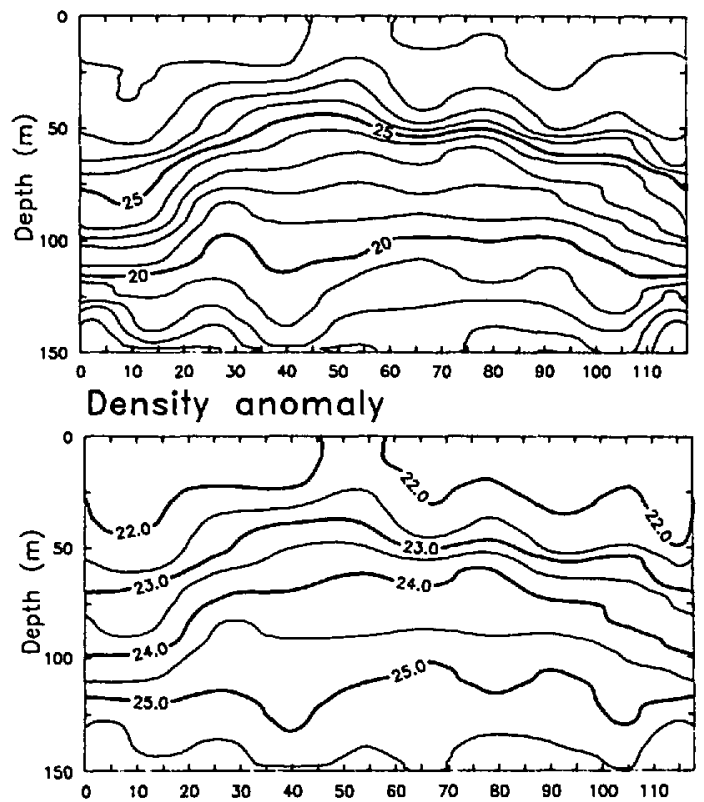

Chlorophyll

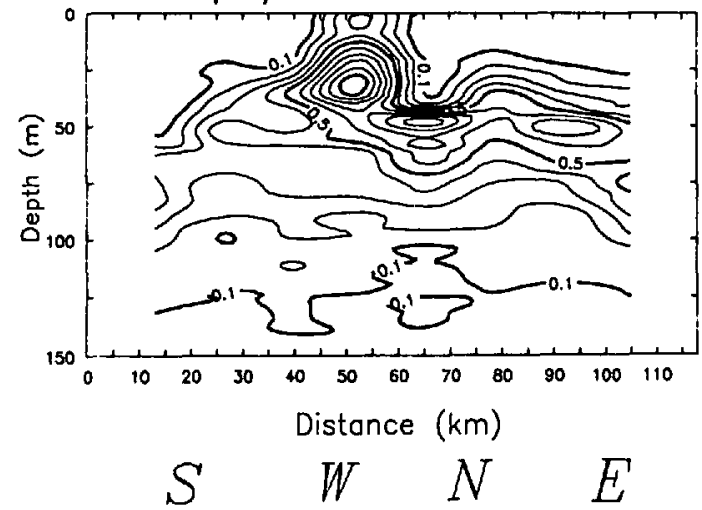

Figure 5. Vertical sections of temperature, density anomaly and chlorophyll ( $\mathrm{mg} \mathrm{m}^{-3}$ ) during the inner circle CTD survey around Aldabra on Cruise 22. The horizontal axis shows distance around the circuit. Points of the compass are marked for orientation relative to the flow (Fig. 4a).

flanks of the island was only $0.5 \mathrm{~m} \mathrm{~s}^{-1}$ and the overall pattern was much less coherent (compare Fig. 4a from Cruise 22). Indeed, the currents to the south of Aldabra appear to be eastward, while to the west current vectors lie in all directions. The sea-surface temperature pattern (Fig. 7b) did not show a convincing cooling in the wake of the island, nor did we observe a coherent patch of more saline water (Fig. 7c) which would have implied some mixing or upwelling of water from below the mixed layer into the 

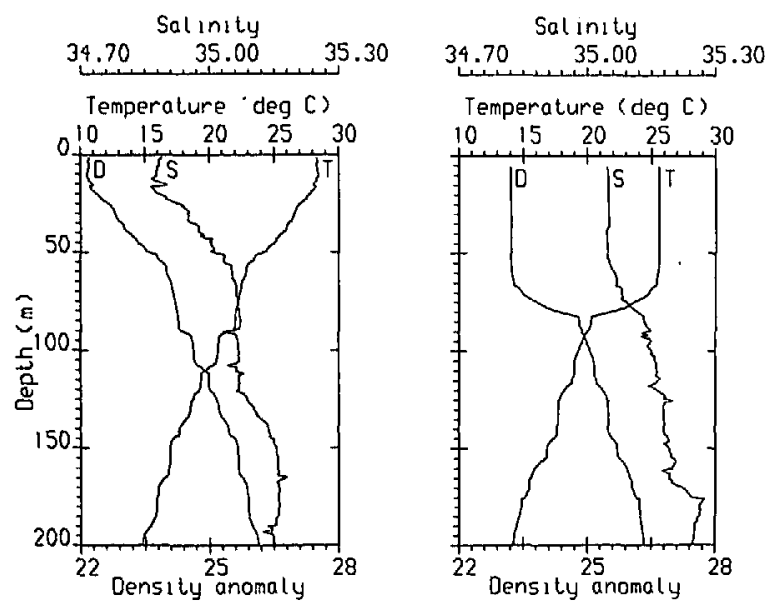

Figure 6. Typical profiles of temperature, density and salinity during the two cruises.

surface water. The integrated chlorophyll distribution (Fig. 7d) showed patches of fairly high chlorophyll $\left(50 \mathrm{mg} \mathrm{m}^{-3}\right)$ both upstream and downstream of the island. Vertical sections showed no significant doming of isopycnals.

c. Surveys of Cosmoledo. The smaller island group, Cosmoledo, was surveyed twice during the first cruise. The first visit took place just after we had experienced a fairly strong current incident on Aldabra. However the currents were weak (only $0.2 \mathrm{~m} \mathrm{~s}^{-1}$ maximum) and there was little coherent variation around the island; although the prevailing current was still from the southeast, in many places the current direction appeared random, and a chaotic picture was observed. The temperature and density sections showed little variation around the island. The isopycnals varied by less than $30 \mathrm{~m}$ in the entire circuit of stations. The largest chlorophyll content occurred on the upstream side. Thus during this period of weak currents, an effect on the isopycnals, SST or chlorophyll was not seen, as happened in similar circumstances for Aldabra in June.

However a return visit to Cosmoledo two weeks later appeared to show a clear island influence on the density structure (Fig. 8). A doming of isopycnals and isotherms was observed in the lee of the islands. For example, the $28^{\circ} \mathrm{C}$ isotherm rose $40 \mathrm{~m}$ from $60 \mathrm{~m}$ in the east to $20 \mathrm{~m}$ in the west. The chlorophyll, confined in the thermocline region, outcropped on the surface at four CTD stations on the northwest flank. At this time the current speed had increased (Fig. 9) compared to the previous visit to Cosmoledo. The overall pattern, although the sampling was sparse, indicated that there was a strong current to the west, which reached a maximum of $1 \mathrm{~m} \mathrm{~s}^{-1}$ on the northern and southern sides of the island. In the lee (to the west of the island), the flow was disordered and weak. The integrated chlorophyll around the circuit of CTD stations varied only 

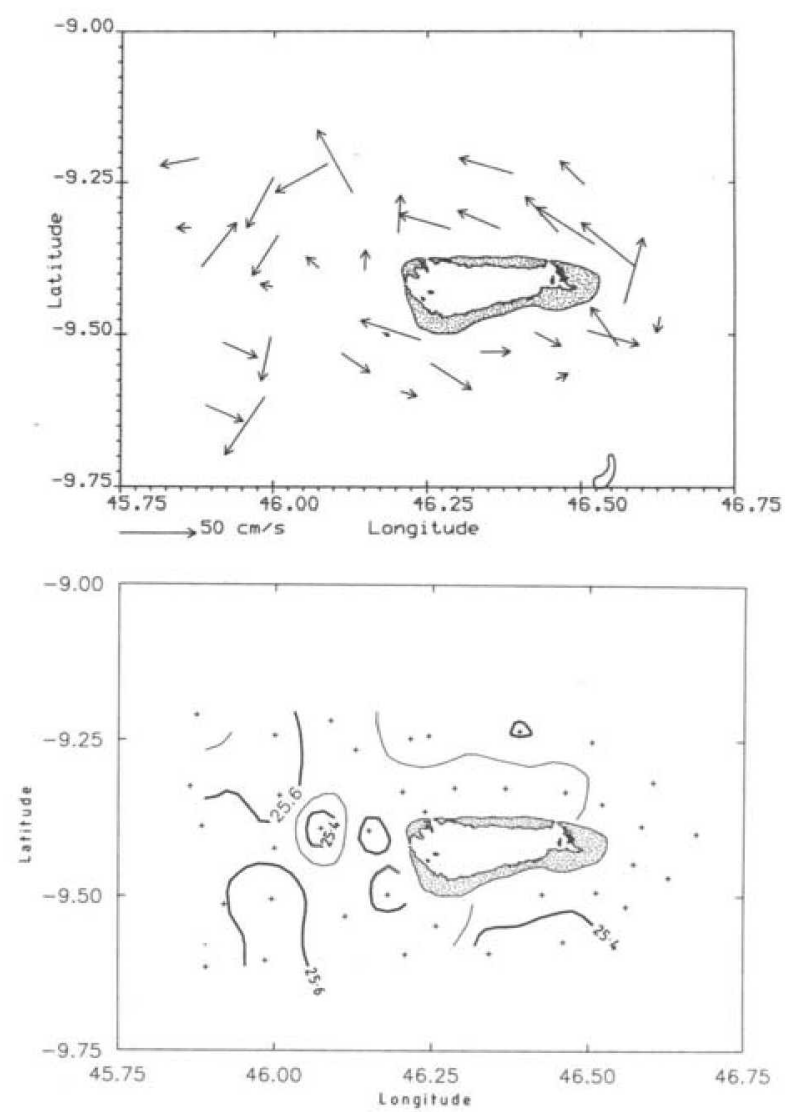

Figure 7. Oceanographic parameters during the CTD survey around Aldabra on Cruise 24, 23 June 1430-27 June 0930. Otherwise as Figure 4.

between $25 \mathrm{mg} \mathrm{m}^{-2}$ and $45 \mathrm{mg} \mathrm{m}^{-2}$; the highest values occurred to the south of Cosmoledo in the area of high current on the flank.

\section{Discussion}

In Section 2 it was shown that the criteria for flow separation and eddy formation depend upon whether the rotation rate is constant across the region of interest ( $f$ plane) or varies with latitude ( $\beta$ plane). The dimensionless parameter $\beta^{\prime}$ determines the importance of the $\beta$ effect. Aldabra is situated at about $10 \mathrm{~S}$, its radius is about $10 \mathrm{~km}$, and incident currents varied between 0.1 and $0.6 \mathrm{~m} \mathrm{~s}^{-1}$. A range of $4 \times 10^{-3}$ to $2 \times$ $10^{-2}$ is obtained for $\beta^{\prime}$; since these are substantially less than unity, it is concluded that the $\beta$ effect is unimportant here and an $f$ plane approximation is adequate. Therefore the results from $f$ plane theory and laboratory experiments may be compared with this data set. 

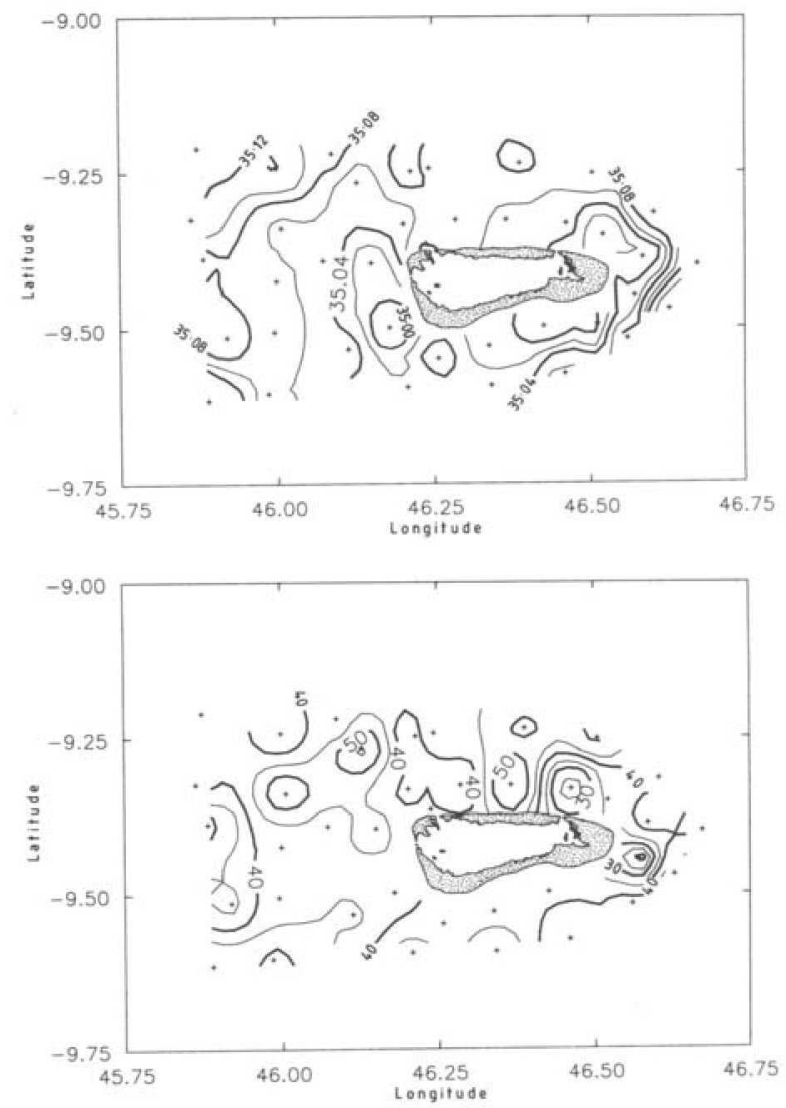

Figure 7. (Continued)

Figure 10 summarizes the regime boundaries found by Baines and Davies (1980) to determine at which values of $R O$ and $E k$ flow separation and eddy formation occur. In geophysical flows, there is considerable uncertainty in calculating $E k$ since the eddy viscosity may vary by several orders of magnitude (Apel, 1987). Barkley (1972) uses an eddy viscosity of $2 \times 10^{2} \mathrm{~m}^{2} \mathrm{~s}^{-1}$ when considering a similar situation to ours, namely the flow around Johnston Atoll in the Pacific. Salusti and Santoleri (1984) and Pattiaratchi et al. (1987) use $\nu=100 \mathrm{~m}^{2} \mathrm{~s}^{-1}$, which we shall apply here.

Rossby, Ekman and Reynolds numbers have been calculated for each of the case studies described earlier, and are given in Table 1. We would emphasize however that these values are very much dependent on the eddy viscosity used.

These values have been plotted on Figure 10. The two higher incident velocity cases lie in the trapped eddy regime, while the two low incident current cases lie near the boundary between fully attached flow and the beginning of eddy formation. These results are similar to those of Barkley (1972). His observations of flow around Johnston 

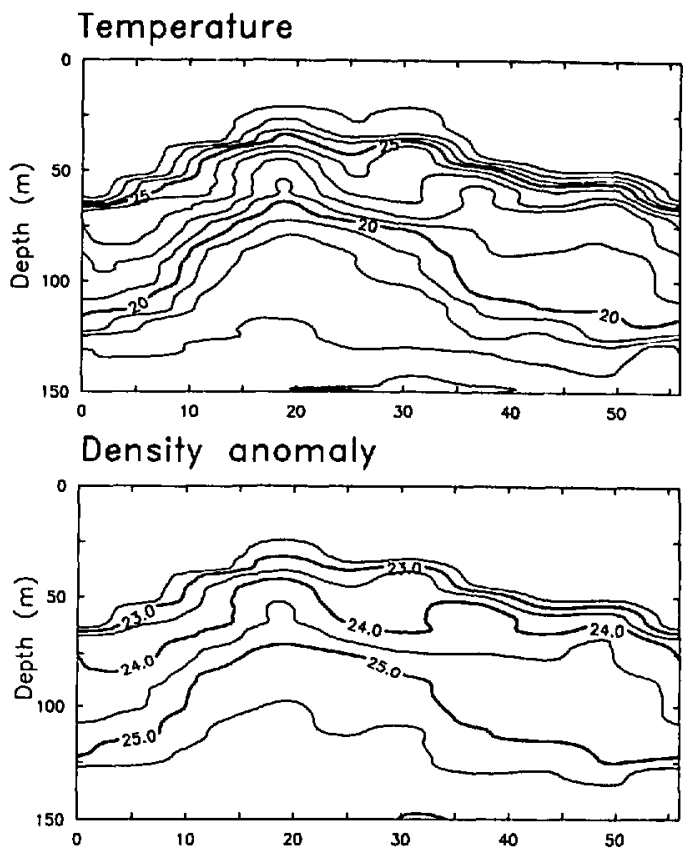

Chlorophyll
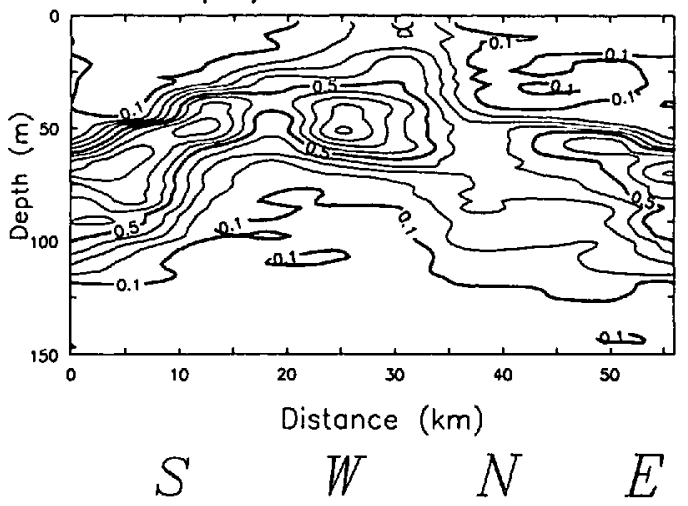

Figure 8. Vertical sections from the second survey around Cosmoledo, showing temperature, density and chlorophyll $\left(\mathrm{mg} \mathrm{m}^{-3}\right)$.

atoll (which has a radius of about $5 \mathrm{~km}$ ) showed that when the incident current was weak $\left(0.15-0.2 \mathrm{~m} \mathrm{~s}^{-1}\right)$ and the Reynolds number only 20 , no wake was evident. However during periods of strong incident flow $\left(0.6 \mathrm{~m} \mathrm{~s}^{-1}\right)$ when the Reynolds number was 70 , a Von Karman vortex street was observed.

In view of the uncertainty in oceanic eddy viscosity $\nu$, it is perhaps fortuitous that the Ekman and Rossby numbers calculated here fell within the trapped eddy regime. However we have additional data which also suggest that the trapped eddy regime is the most appropriate during the case studies discussed here. We made several ADCP 


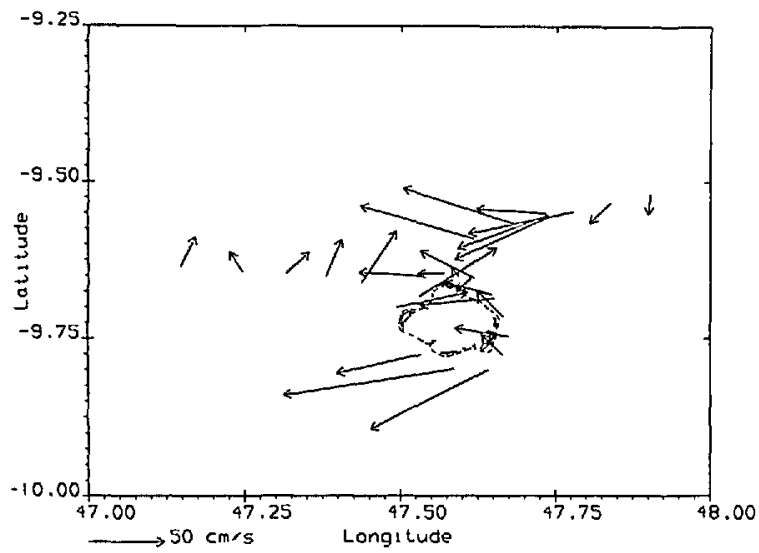

Figure 9. Half-hour averages of $19 \mathrm{~m}$ ADCP currents, 2 May 1700-3 May 1800 during the second survey around Cosmoledo. The dashed line shows the outer edge of the reef, normally covered except at low water.

runs across and along the region where a wake would have been expected, but none was observed. The current meters do not suggest eddies at these times. Additional CTD surveys do not point to anomalous regions of lower SST and higher chlorophyll travelling down a wake. If we invert the problem, and make the assumption that a trapped eddy regime is applicable, then we can use the regime diagram (Fig. 10) to give bounds on the eddy viscosity. For the stronger current around Aldabra, this produces a range of 30 to $150 \mathrm{~m}^{2} \mathrm{~s}^{-1}$ for $\nu$.

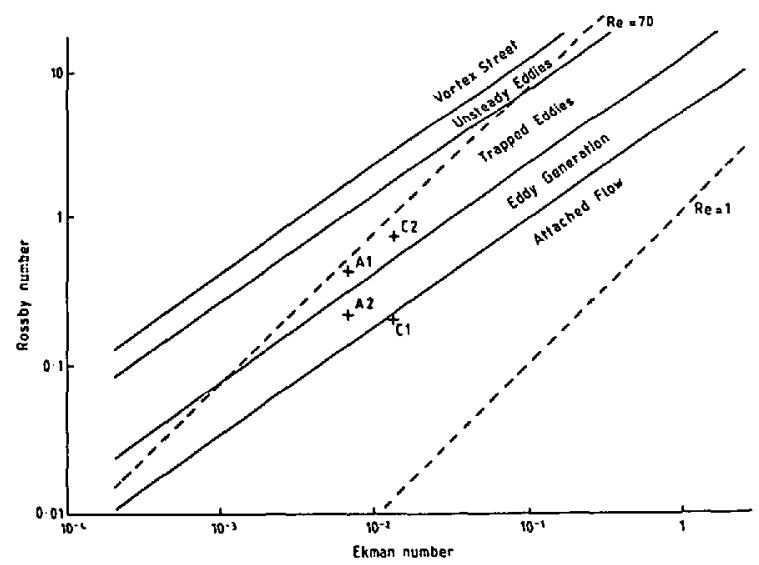

Figure 10. Rossby number/Ekman number flow regime diagram showing the regime boundaries from Baines and Davies (1980) and the values for the four case studies discussed here. The dashed lines indicate Reynolds numbers of 1 and 70, the "traditional" thresholds for attached flow and vortex streets. 
Table 1.

\begin{tabular}{lccccr}
\multicolumn{1}{c}{ Island } & $\begin{array}{c}\text { Radius } \\
(\mathrm{m})\end{array}$ & $\begin{array}{c}\text { Current } \\
\left(\mathrm{m} \mathrm{s}^{-1}\right)\end{array}$ & $R o$ & $E k$ & $R e$ \\
Aldabra & $10^{4}$ & 0.6 & 0.41 & $6.9 \times 10^{-3}$ & 59 \\
Aldabra & $10^{4}$ & 0.3 & 0.21 & $6.9 \times 10^{-3}$ & 30 \\
Cosmoledo & $7 \times 10^{3}$ & 0.2 & 0.20 & $1.4 \times 10^{-2}$ & 14 \\
Cosmoledo & $7 \times 10^{3}$ & 0.7 & 0.69 & $1.4 \times 10^{-2}$ & 49
\end{tabular}

In summary, the flow regime diagram (Fig. 10) indicates little disturbance to the flow in cases where the incident flow is weak, only of the order of 0.2 to $0.3 \mathrm{~m} \mathrm{~s}^{-1}$. During periods of steady stronger flow, however (greater than $\sim 0.5 \mathrm{~m} \mathrm{~s}^{-1}$ ), one or more eddies are trapped in the lee of the island. Since Aldabra is in the southern hemisphere, an enhancement of clockwise eddies would be expected. Had the incident current been greater than $1 \mathrm{~m} \mathrm{~s}^{-1}$ as climatological data suggested (Crepon, 1964; Cutler and Swallow, 1984) and relatively constant in direction, a vortex street of eddies should have been spun off behind the islands of Aldabra and Cosmoledo, since in this case the Reynolds number would be about 100 .

Therefore in two of the case studies (Figs. 4 and 9) one might expect to see evidence of a trapped clockwise eddy. The ADCP data (Fig. 4a) are really too sparse to confirm this, although it is encouraging that dynamic topography from the CTD surveys also suggests a clockwise eddy. Lack of a systematic pattern in the ADCP currents may be due to the nonsynopticity of the observations. The CTD surveys took up to three days to complete, during which time some variation in the flow is to be expected since the system is not in local steady state.

During periods of weak $\left(<0.5 \mathrm{~m} \mathrm{~s}^{-1}\right)$, variable (changing by $90^{\circ}$ in 3 days) far field currents (Fig. 7), the islands do not seem to have a significant effect on the hydrography; the changes in isopycnal depth around the island are small and random and are probably due to internal waves and tides. There are no apparent correlations between the variations in isopycnal depth and chlorophyll concentration. The distribution of phytoplankton during these periods is indicative of a general patchiness.

Our two case studies where the far-field flow is both steady (constant for several days) and strong (greater than $0.5 \mathrm{~m} \mathrm{~s}^{-1}$ ) do show an effect of the island topography on the observed hydrography. The principal hydrographic effect is a doming of the isopycnals immediately behind the island; a near-surface isopycnal may rise by more than $50 \mathrm{~m}$. This effect on density is mainly associated with a cooler temperature behind the island at depths from the surface to about $100 \mathrm{~m}$. There is also evidence for higher salinity water occurring in the lee. These results suggest upwelling of cooler, saltier water which may be associated with a trapped cyclonic eddy behind the island. Alternatively, or in addition, there may be enhanced mixing happening near the surface, either behind the island, or on the flanks and being advected into the lee.

Previous work on the effect of topography in shallow water (Simpson and Tett, 1986) 
has shown that such enhanced mixing on the flanks of an island leads to a greater supply of nutrients in near-surface waters, and hence to a greater abundance of phytoplankton (the "island mass effect"). The first CTD survey of Cruise 22 (Fig. 4) shows considerably higher chlorophyll in the surface waters behind the island on both inner and outer circuits ( $70 \mathrm{mg} \mathrm{m}^{-3}$ compared to a background value of $30 \mathrm{mg} \mathrm{m}^{-3}$ ). This correlates well with the region of lower SST, and of weaker and fluctuating currents.

It should be noted that these effects cannot be caused by tidal flushing of the Aldabra lagoon for several reasons. First, the increased chlorophyll region is associated with cooler water, whereas the lagoon water would be warmer than the surroundings (Pugh and Rayner, 1981). Second, even if the entire lagoon water were exchanged each tidal cycle, the volume of water could not cause the vertical and horizontal coverage of enhanced productivity that was observed. Finally, a similar doming of isopycnals is observed at Cosmoledo. The cool, high productivity water must be associated with a mixing of nutrient-rich water from below the thermocline.

\section{Conclusions}

Despite great interest in the topic of flow disturbance by cylinders or islands in the fluid mechanics literature, there are few observational data sets to test the applicability of such models and laboratory experiments to the real ocean. Recently developed instrumentation such as the ADCP has enabled us to describe the flow around two small (radius $\sim 10 \mathrm{~km}$ ) islands in an ocean current. The results are consistent with theory, in that at moderate speeds an eddy appeared trapped in the lee of the island, while at lower speeds no eddy activity was observed. The current speeds were too low to expect a wake of eddies to be spun off behind the island.

On those occasions where an eddy did appear trapped behind Aldabra, a significant effect was observed on the hydrography and biology. The isopycnals rose in the lee, giving an area of colder sea-surface temperature, associated with enhanced chlorophyll concentration due to greater phytoplankton productivity. Cooler water has been brought from below the surface, bringing with it nutrients which increase productivity. Therefore if an oceanic island is in a region of strong, steady current, the island mass effect is an important contribution to the productivity and potential for fisheries in the area.

Acknowledgments. The authors would like to thank all those associated with Cruises 22 and 24 of the RRS Charles Darwin for their hard work and enthusiasm which made the experiment both successful and enjoyable. We are grateful to Paul Tett for his painstaking calibration of the CTD fluorometer for chlorophyll concentration, to Ron Haynes for the CTD processing software, to Paddy Dewes for drawing Figures 1 and 10, and to Ed Hill for constructive comment on the manuscript. Many thanks to John Collie and Hugh Bernard of the Aldabra Research Station for providing meteorological records during the period of study. This project was supported by NERC grant GR3/5549. 


\section{REFERENCES}

Apel, J. R. 1987. Principles of Ocean Physics, Academic Press, 631 pp.

Baines, P. G. and P. A. Davies. 1980. Laboratory studies of topographic effects in rotating and/or stratified fluids, in Orographic Effects in Planetary Flows, R. Hide and P. White, eds., GARP Publ. Ser., 23, WMO, Geneva. 233-299.

Barkley, R. A. 1972. Johnston Atoll's wake. J. Mar. Res., 30, 201-216.

Batchelor, G. K. 1967. An Introduction to Fluid Dynamics, Cambridge Univ. Press, 515 pp.

Boyer, D. L. and P. A. Davies. 1982. Flow past a circular cylinder on a $\beta$ - plane. Phil. Trans. R. Soc. Lond., A306, 533-556.

Crepon, M. 1964. Presentation d'observations faites au GEK en 1962, Cahiers Oceanographiques, XVI, 10.

Cutler, A. N. and J. C. Swallow. 1984. Surface currents of the Indian Ocean (to 25S, 100E): compiled from historical data archived by the Meteorological Office, Bracknell, U.K., Institute of Oceanographic Sciences, Report No. 187, 8 pp \& 36 charts.

Doty, M. S. and M. Oguri. 1956. The island mass effect. J. Conseil Perm. Int. Ex. Mer., 22. 33-37.

FAO. 1981. Report of the Committee for Development and Management of Fisheries in the Southwest Indian Ocean, FAO Fisheries Report 254.

Fett, R. W. and K. M. Rabe. 1976. Island barrier effects on sea state as revealed by a numerical wave model and DMSP satellite data. J. Phys. Oceanogr., 6, 324-334.

Gilmartin, M. and N. Revelante. 1974. The island mass effect on the phytoplankton and primary production of the Hawaiian islands. J. Exp. Mar. Biol. Ecol., 16, 181-204.

Gordon, H. B. and R. L. Hughes. 1981. A study of rotating baroclinic nonlinear flow around an island. J. Phys. Oceanogr., 11, 1011-1014.

Hogg, N. G. 1972. Steady flow past an island with application to Bermuda. Geophys. Fluid Dyn. 4. 55-81.

- 1980a. Effects of bottom topography on ocean currents, in Orographic Effects in Planetary Flows, R. Hide and P. White, eds., GARP Publ. Ser., 23, WMO, Geneva, 167-205.

1980b. Observations of internal Kelvin waves trapped round Bermuda. J. Phys. Oceanogr., $10,1353-1376$.

Hogg, N. G., E. J. Katz and T. B. Sandford. 1978. Eddies, islands and mixing. J. Geophys. Res., 83, 2921-2938.

La Violette, P. E. 1974. A satellite-aircraft thermal study of the upwelled waters off Spanish Sahara. J. Phys. Oceanogr., 4, 676-684.

Merkine, L. O. 1980. Flow separation on a $\beta$ plane. J. Fluid Mech., 99, 399-409.

Pattiaratchi, C., A. James and M. Collins. 1987. Island wakes and headland eddies: a comparison between remotely sensed data and laboratory experiments. J. Geophys. Res., 92(C1), 783-794.

Pollard, R. T. and J. Read. 1989. A method for calibrating shipmounted Acoustic Doppler Profilers, and the limitations of gyro compasses. J. Atmos. Oceanic Tech., (in press).

Pugh, D. T. and R. F. Rayner. 1981. The tidal regimes of three Indian Ocean atolls and some ecological implications. Estuar., Coastal Shelf Sci., 13, 389-407.

Salusti, E. and R. Santoleri. 1984. A von Karman wake in the Ligurian Sea. Boll. Oceanol. Teor. Appl., 2, 275-279.

Simpson, J. H. and P. B. Tett. 1986. Island stirring effects on phytoplankton growth, in Lecture Notes on Coastal and Estuarine Studies, 17. Tidal Mixing and Plankton Dynamics, J. Bowman, M. Yentsch and W. T. Peterson, eds., Springer-Verlag, Berlin Heidelberg, 41-76.

Simpson, J. H., P. B. Tett, M. L. Argote-Espinoza, A. Edwards, K. J. Jones and G. Savidge. 
1982. Mixing and phytoplankton growth around an island in a stratified sea. Cont. Shelf Res., $1,15-31$.

Takahashi, M., Y. Yasuoka, M. Watanabe, T. Miyazaki and S. Ichimura. 1981. Local upwelling associated with vortex motion off Oshima Island, in Coastal Upwelling, F. Richards, ed., A.G.U., Washington, DC.

Tett, P. B. 1990. In situ fluorometric measurement of tropical oceanic chlorophyll, (manuscript in preparation).

Wang, D. P. 1982. Development of a three-dimensional, limited-area (island) shelf circulation model. J. Phys. Oceanogr., 12,605-617.

White, W. B. 1973. An oceanic wake in the equatorial undercurrent downstream from the Galapagos Archipelago. J. Phys. Oceanogr., 3, 156-161.

Received: 10 July, 1989; revised: 19 October, 1989. 